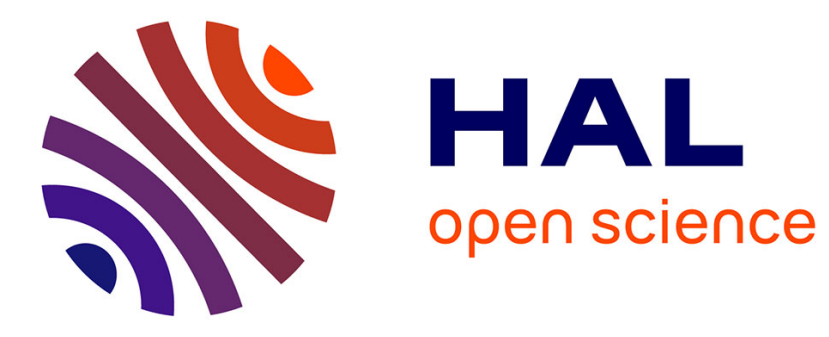

\title{
Design and characterization of extreme-ultraviolet broadband mirrors for attosecond science
}

\author{
Anne-Sophie Morlens, Rodrigo B. Lopez-Martens, Olga Boyko, Philippe \\ Zeitoun, Philippe Balcou, Katalin Varjú, Erik Gustafsson, Thomas Remetter, \\ Anne L'Huillier, Sophie Kazamias, et al.
}

\section{To cite this version:}

Anne-Sophie Morlens, Rodrigo B. Lopez-Martens, Olga Boyko, Philippe Zeitoun, Philippe Balcou, et al.. Design and characterization of extreme-ultraviolet broadband mirrors for attosecond science. Optics Letters, 2006, 31 (10), pp.1558-1560. 10.1364/OL.31.001558 . hal-00521042

HAL Id: hal-00521042

https://hal-polytechnique.archives-ouvertes.fr/hal-00521042

Submitted on 23 Mar 2012

HAL is a multi-disciplinary open access archive for the deposit and dissemination of scientific research documents, whether they are published or not. The documents may come from teaching and research institutions in France or abroad, or from public or private research centers.
L'archive ouverte pluridisciplinaire HAL, est destinée au dépôt et à la diffusion de documents scientifiques de niveau recherche, publiés ou non, émanant des établissements d'enseignement et de recherche français ou étrangers, des laboratoires publics ou privés. 


\title{
Design and characterization of extreme-ultraviolet broadband mirrors for attosecond science
}

\author{
Anne-Sophie Morlens, Rodrigo López-Martens, Olga Boyko, Philippe Zeitoun, and Philippe Balcou \\ Laboratoire d'Optique Appliquée, Ecole Nationale Supérieure des Techniques Avancées-Ecole Polytechnique- \\ CNRS UMR 7639, F-91761 Palaiseau, France
}

Katalin Varjú, Erik Gustafsson, Thomas Remetter, and Anne L'Huillier

Department of Physics, Lund University, P.O. Box 118, 22100 Lund, Sweden

Sophie Kazamias

Laboratoire d'Interaction du rayonnement X Avec la Matière, Université Paris-Sud, 91405 Orsay, France

Julien Gautier, Franck Delmotte, and Marie-Françoise Ravet

Laboratoire Charles Fabry de l'Institut d'Optique, CNRS-UMR 8501, 91403 Orsay, France

\begin{abstract}
Received November 29, 2005; accepted December 21, 2005; posted February 23, 2006 (Doc. ID 66345)
A novel multilayer mirror was designed and fabricated based on a recently developed three-material technology aimed both at reaching reflectivities of about $20 \%$ and at controlling dispersion over a bandwidth covering photon energies between 35 and $50 \mathrm{eV}$. The spectral phase upon reflection was retrieved by measuring interferences in a two-color ionization process using high-order harmonics produced from a titanium: sapphire laser. We demonstrate the feasibility of designing and characterizing phase-controlled broadband optics in the extreme-ultraviolet domain, which should facilitate the manipulation of attosecond pulses for applications. (C) 2006 Optical Society of America

OCIS codes: $120.5050,230.4170,320.7160,340.7470$.
\end{abstract}

The technology of x-ray and extreme ultraviolet (XUV) ultrashort light sources has made tremendous progress during the past few years, thus opening up a whole range of possibilities for investigating problems in material and life sciences. This leap forward in XUV light sources requires stringent concomitant progress in dedicated optical instrumentation, starting from the simplest and most ubiquitous elements: mirrors. The simple transport of attosecond XUV pulses $^{1,2}$ is difficult, since the spectral phase has to be maintained over spectral bandwidths spanning tens of electron volts. Moreover, it has been shown that attosecond pulses obtained from laser-driven highharmonic generation sources often present an intrinsic chirp that has to be compensated for in order to obtain ultrashort light pulses. ${ }^{3,4}$ Aperiodic multilayer mirror technology has been suggested to control dispersion in the XUV domain. ${ }^{5,6}$ Reflection off a mirror surface can be described as the product of the spectral amplitude $E(\omega)$ of a light pulse and the reflection coefficient, $\quad r(\omega)=\sqrt{R(\omega)} \exp [i \varphi(\omega)]$, of the mirror, which includes an amplitude and a phase (Fig. 1). The design of broadband mirrors implies characterizing both the reflectance $R(\omega)$ and the phase $\varphi(\omega)$ or, equivalently, the phase variation $\partial \varphi / \partial \omega$ over the mirror spectral range. In this Letter we report the characterization of both the reflectance and the phase of a broadband XUV mirror with high reflectivity and phase control for photon energies around $40 \mathrm{eV}$.

The multilayer mirror used in this study represents a step toward aperiodic multilayer mirrors, ${ }^{6}$ albeit with a simpler design. ${ }^{7}$ The mirror was developed for the spectral region around $40 \mathrm{eV}$, for which the conversion efficiency for high-order harmonic generation in argon reaches high values, and which is therefore particularly well suited to applications in attosecond metrology. While standard mirrors around $40 \mathrm{eV}$ consist of periodic stacks of only two materials, such as $\mathrm{Mo} / \mathrm{Si}$ or $\mathrm{B}_{4} \mathrm{C} / \mathrm{Si}$, here we added a third material in the period of the multilayer, which now consists of $\mathrm{Mo} / \mathrm{Si} / \mathrm{B}_{4} \mathrm{C}{ }^{8}$ As shown in Fig. 1, we superimposed several periods (2 and 4 ) of two different multilayers, with thickness 16.3 and $40.6 \mathrm{~nm}$, leading to reflectivity maxima at $40 \mathrm{eV}$ for the thinnest and 33 and $50 \mathrm{eV}$ for the thickest. This allows us to achieve almost $20 \%$ reflectivity over a $20 \mathrm{eV}$ bandwidth centered around $42 \mathrm{eV}$. The mirror tested here was specifically designed for optimizing the reflectivity over a large bandwidth while keeping a constant phase variation. The choice of design was guided by numerical simulations using refractive index data. ${ }^{9}$

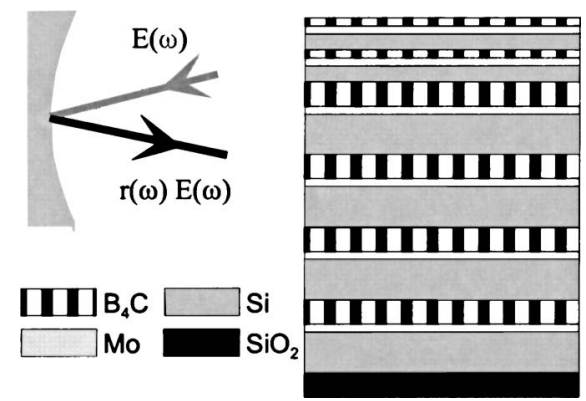

Fig. 1. Aperiodic multilayer structure of the threematerial broadband XUV mirror. From the top to the bottom, there are two $16.3 \mathrm{~nm}$ thick and four $40.6 \mathrm{~nm}$ thick $\mathrm{B}_{4} \mathrm{C} / \mathrm{Mo} / \mathrm{Si}$ layers sitting on a $\mathrm{SiO}_{2}$ substrate. 

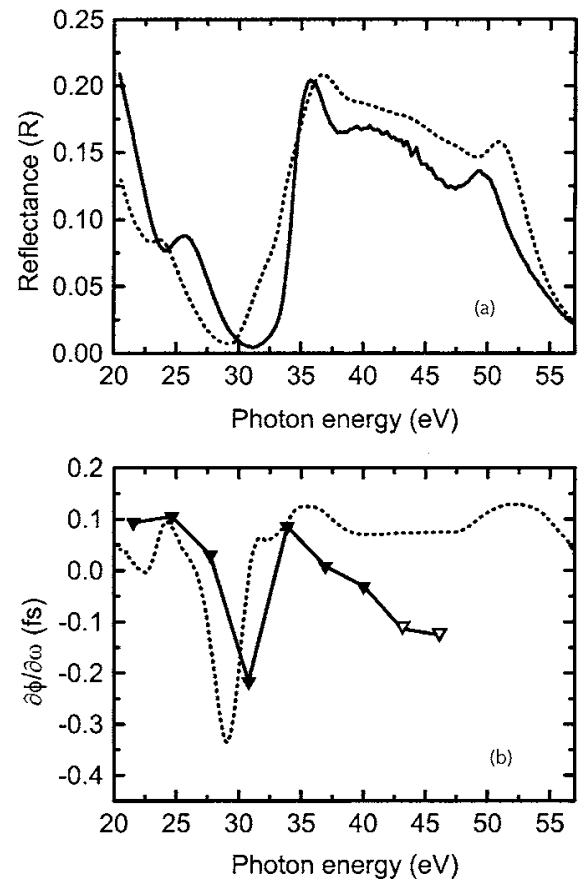

Fig. 2. (a) Synchrotron-based reflectance measurement (solid curve) and calculated reflectance (dashed curve) of the broadband XUV mirror. (b) Phase variation (group delay) of the broadband XUV mirror obtained from harmonic phase measurements (solid curve; see text for details) and calculations (dashed curve).

The results of these simulations for the present design are shown by dotted curves in Fig. 2 . By varying the number of periods and the thickness of the multilayers, custom-made phase variations and reflectances are possible.

Figure 2(a) (solid curve) shows experimental measurements of the reflectance of our multilayer mirror performed at the BEAR beam line of the ELETTRA synchrotron radiation source. The observed wavelength shift between experiment and theory, around $30 \mathrm{eV}$, is most probably due to uncertainties in the materials refraction indices. The lower experimental reflectance, especially at high energy, might come from underestimating the surface roughness of the deposited material. (In the calculations we use a roughness estimated to $0.5 \mathrm{~nm}$.) The same uncertainties will affect the spectral phase response of the mirror; experimental methods should therefore be used to characterize the mirror amplitude and phase responses to optimize its design for a given application.

To measure the phase variation induced by reflection, we used a high-order harmonic source developed at the Lund Laser Center, devoted to the characterization and application of XUV attosecond pulses. ${ }^{10,11}$ The experimental setup is depicted in Fig. 3. Harmonics $13-31$ of an $800 \mathrm{~nm} 1 \mathrm{kHz}$ titanium:sapphire laser were used to span at discrete frequencies part of the spectral range of the mirror. The phase measurements were carried out by using the same technique that is used to characterize the temporal shape of attosecond radiation, called RABITT ${ }^{1,11}$ (reconstruction of attosecond beating by interference of two-photon transitions). A $40 \mathrm{fs}, 810 \mathrm{~nm}$ pulse is sent into an attosecond pump-probe optical delay line.
The pump beam is focused into a $3 \mathrm{~mm}$ static argon gas cell $(30 \mathrm{hPa})$ with a $50 \mathrm{~cm}$ spherical mirror to generate odd high harmonics. The generated harmonics are first separated from the laser light by a $200 \mathrm{~nm}$ thick aluminum filter, then pass through a $1.5 \mathrm{~mm}$ hole in the middle of a spherical mirror designed to match the divergence of the XUV and probe IR beams. Both are then focused, either at normal incidence by the $20 \mathrm{~cm}$ focal length spherical multilayer mirror or at grazing incidence by a platinum-coated mirror (not shown), into the sensitive region of a magnetic bottle electron time-of-flight spectrometer, filled with a rare gas at a few $10^{-4}$ of hectopascals of static pressure. Photoionization of the rare gas by the XUV radiation leads to time-of-flight electron spectra that depend on the pump-probe delay. Absorption of the odd-order high-harmonic photons yields discrete peaks in the spectra, separated by twice the laser photon energy; the probe field induces additional absorption or emission of IR photons, appearing as sidebands. Each sideband has contributions from two neighboring harmonics and contains information about their relative phases. The sideband intensity is proportional to $\cos \left(\Delta \phi_{q+1}\right.$ $\left.+2 \omega_{0} \tau\right)$, where $\Delta \phi_{q+1}=\phi_{q+2}-\phi_{q}, q$ and $q+2$ being two consecutive (odd) harmonic orders, $\omega_{0}$ the laser frequency, and $\tau$ the delay between the XUV and IR fields. The measured $\Delta \phi_{q+1}$ is the sum of four contributions:

$$
\Delta \phi_{q+1}=\Delta \phi_{q+1}^{\mathrm{harm}}+\Delta \phi_{q+1}^{\mathrm{atom}}+\Delta \phi_{q+1}^{\mathrm{Al}}+\Delta \phi_{q+1}^{\text {mirror }},
$$

corresponding, respectively, to the intrinsic chirp of the harmonic generation process, ${ }^{3,4}$ the chirp of the photoionization process ${ }^{12}$ (negligible here), the groupdelay dispersion of the aluminum filter, ${ }^{11}$ and the chirp induced by the focusing mirror.

Our characterization method consists in studying the sideband signal as a function of pump-probe delay over a large spectral range. $\Delta \phi_{q+1}$ as a function of order $q$ is then obtained from the phase of the sideband oscillations. The results are shown in Fig. 4 as the dashed curve for the measurements with the grazing-incidence platinum-coated mirror and as the solid curve for the multilayer mirror.

In the case of the platinum mirror, the phase distortion due to metallic reflection is negligible, and the observed phase variation comes essentially from the

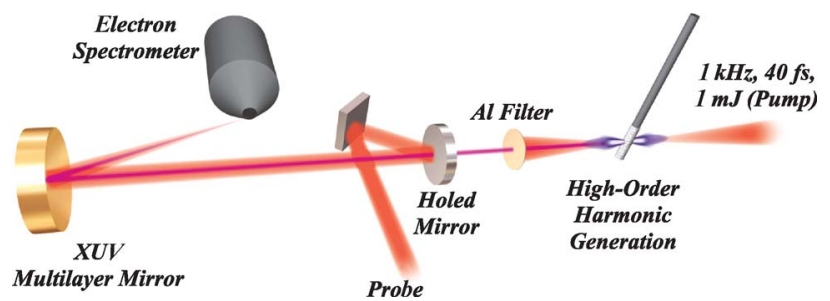

Fig. 3. (Color online) Experimental setup used for characterizing the phase variation induced by broadband XUV multilayer mirrors. The measurement is based on reconstructing attosecond pulse trains synthesized in a laserdriven high-harmonic source and reflected off the mirror. The multilayer structure was deposited on a spherical mirror substrate with a $20 \mathrm{~cm}$ focal length. 


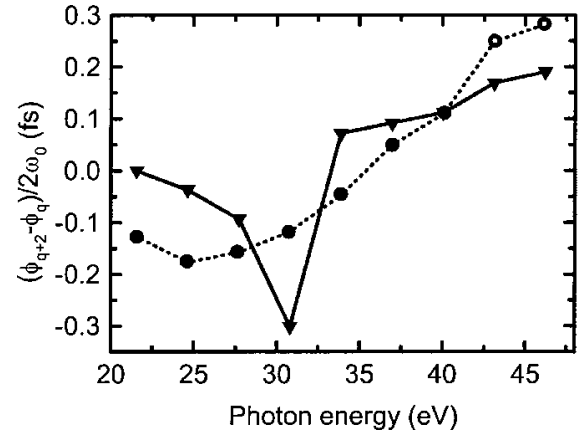

Fig. 4. Phase variation measurements performed by using a platinum-coated grazing-incidence mirror (dashed curve) and the broadband XUV mirror at normal incidence (solid curve).

intrinsic positive attosecond chirp of the harmonics, ${ }^{3,4}$ with a slight contribution from the negatively dispersive aluminum filter. ${ }^{10}$ The electron signals for harmonics 29 and 31 were very low and could not be used to measure the sideband phase oscillation. For these last two points the harmonic phases were extrapolated by solving the saddle point equations within the strong-field approximation. ${ }^{13}$ The results with the multilayer mirror exhibit rapid phase variations, especially around $30 \mathrm{eV}$. By substracting the results obtained with both mirrors, we deduced the effect of the multilayer mirror on the phase variation, $\partial \varphi / \partial \omega \simeq \Delta \phi_{q+1}^{\text {mirror }} / 2 \omega_{0}$, as indicated by the solid curve in Fig. 2(b). Remarkably, the discrepancies between experimental results and simulations are quite close to those shown for the reflectance in Fig. 2(a). The rapid variation around $30 \mathrm{eV}$ corresponds to the passage from total surface reflection to interferential reflection for higher photon energies. The full mirror range could not be explored because of the low high-harmonic spectral cutoff energy in argon.

Within the spectral range of the mirror that we could sample, the negative dispersion induced by the multilayer mirror reduces the intrinsic chirp of the harmonic radiation, in principle making it possible to temporally compress the reflected attosecond pulses. Unfortunately, the relatively high reflectivity for the low-order harmonics, combined with the phase jump around $30 \mathrm{eV}$, prevent significant compression of the attosecond pulses below 250 as. The high reflectivity in the lower photon energy region (below $30 \mathrm{eV}$ ) could be significantly reduced by using filters combining aluminum and silicon materials. Neon could also be used to generate harmonics over a broad spectral range spanning the whole optimized region of the multilayer, thus achieving pulse compression below 150 as with optimized phase compensation.

In summary, we have designed and characterized a new multilayer mirror with broad reflectance around $40 \mathrm{eV}$ featuring custom dispersion control to compress attosecond XUV pulses. To the best of our knowledge, this the first time a broadband phase measurement has been performed in the notoriously difficult XUV wavelength region. These findings reveal that, despite the current uncertainties in material properties such as refractive index, deposited density, and surface roughness, dispersion control of ultrashort light pulses can be extended from the visible-infrared to the XUV-soft-x-ray spectral range. Such instrumentation is crucial for shaping and transporting attosecond pulses over a large and tunable energy range.

This research was supported by a Marie Curie Intra-European Fellowship (MEIF-CT-2004-009268), the Marie Curie Research Training Network XTRA (MRTN-CT-2003-505138), the Integrated Infrastructure Initiative Laserlab-Europe (RII3-CT-2003506350), the Knut and Alice Wallenberg Foundation, and the Swedish Science Council. K. Varjú is on leave from the Department of Optics and Quantum Electronics, University of Szeged, Hungary. A.-S. Morlens's e-mail address is anne-sophie.morlens @ensta.fr.

\section{References}

1. P. M. Paul, E. S. Toma, P. Breger, G. Mullot, F. Augé, Ph. Balcou, H. G. Muller, and P. Agostini, Science 292, 1689 (2001).

2. R. Kienberger, E. Goulielmakis, M. Uiberacker, A. Baltŭska, V. Yakovlev, F. Bammer, A. Scrinzi, Th. Westerwalbesloh, U. Kleineberg, U. Heinzmann, M. Drescher, and F. Krausz, Nature 427, 817 (2004).

3. S. Kazamias and Ph. Balcou, Phys. Rev. A 69, 063416 (2004).

4. Y. Mairesse, A. de Bohan, L. J. Frasinski, H. Merdji, L. C. Dinu, P. Monchicourt, P. Breger, M. Kovačev, R. Taïeb, B. Carré, H. G. Muller, P. Agostini, and P. Salières, Science 302, 1540 (2003).

5. W. Wonisch, Th. Westerwalbesloh, W. Hachmann, K. Kabachnik, U. Kleineberg, and U. Heinzmann, Thin Solid Films 464, 473 (2004).

6. A.-S. Morlens, Ph. Balcou, Ph. Zeitoun, C. Valentin, V. Laude, and S. Kazamias, Opt. Lett. 32, 1540 (2005).

7. F. Delmotte, J. Gautier, M. Roulliay, M. F. Ravet, F. Bridou, and A. Jerome, in Proc. SPIE 5963, 539 (2005).

8. J. Gautier, F. Delmotte, M. Roulliay, F. Bridou, M.-F. Ravet, and A. Jérome, Appl. Opt. 44, 384 (2005).

9. D. Windt, "IMD code," www.bell-labs.com/project/imd/.

10. R. López-Martens, K. Varjú, P. Johnsson, J. Mauritsson, Y. Mairesse, P. Salières, M. B. Gaarde, K. J. Schafer, A. Persson, S. Svanberg, C.-G. Wahlström, and A. L'Huillier, Phys. Rev. Lett. 94, 063901 (2005).

11. K. Varjú, P. Johnsson, R. López-Martens, T. Remetter, E. Gustafsson, J. Mauritsson, M. B. Gaarde, K. J. Schafer, C. Erny, I. Sola, A. Zaïr, E. Constant, E. Cormier, E. Mével, and A. L'Huillier, Laser Phys. 15, 888 (2005).

12. J. Mauritsson, M. B. Gaarde, and K. J. Schafer, Phys. Rev. A 72, 013401 (2005).

13. K. Varjú, Y. Mairesse, B. Carré, M. B. Gaarde, P. Johnsson, S. Kazamias, R. López-Martens, J. Mauritsson, K. J. Schafer, Ph. Balcou, A. L'Huillier, and P. Salières, J. Mod. Opt. 52, 379 (2005). 